\title{
Patterns of radiotherapy practice for pancreatic cancer: Results of the Gastrointestinal Radiation Oncology Study Group multi-institutional survey
}

\author{
GABRIELLA MACCHIA $^{1}$, ALDO SAINATO ${ }^{2}$, RENATO TALAMINI ${ }^{3}$, GIOVANNI BOZ ${ }^{4}$, \\ ALMALINA BACIGALUPO $^{5}$, LUCIANA CARAVATTA ${ }^{6}$, MICHELE FIORE $^{7}$, MARIA LUISA FRISO $^{8}$, \\ VINCENZO FUSCO ${ }^{9}$, MARCO LUPATTELLI ${ }^{10}$, GIOVANNA MANTELLO ${ }^{11}$, GIAN CARLO MATTIUCCI ${ }^{12}$, \\ NAJLA SLIM $^{13}$, PIERA SCIACERO ${ }^{14}$, LUCIA TURRI $^{15}$, VINCENZO VALENTINI $^{12}$, \\ ALESSIO GIUSEPPE MORGANTI ${ }^{1,16^{*}}$ and DOMENICO GENOVESI ${ }^{17^{*}}$
}

${ }^{1}$ Radiation Oncology Unit, Research and Care Foundation 'Giovanni Paolo II', Catholic University of Sacred Heart, Campobasso; ${ }^{2}$ Radiation Oncology Unit, University Hospital, Pisa; ${ }^{3}$ Epidemiology and Biostatistics Unit, and ${ }^{4}$ Radiation Oncology Department, Oncological Referral Center, Aviano; ${ }^{5}$ Radiation Oncology Unit, AOU IRCCS San Martino, IST National Cancer Research Institute, Genoa; ${ }^{6}$ Radiation Oncology Department, 'A. Businco' Regional Oncological Hospital, Cagliari; ${ }^{7}$ Radiation Oncology Unit, Campus Bio-Medico University Hospital, Rome;

${ }^{8}$ Radiotherapy and Nuclear Medicine Unit, Veneto Institute of Oncology-IRCCS, Padua; ${ }^{9}$ Radiation Oncology Unit, IRCCS CROB, Rionero in Vulture, Potenza; ${ }^{10}$ Radiation Oncology Unit, 'S. Maria della Misericordia' Hospital, Perugia;

${ }^{11}$ Radiation Oncology Unit, State Hospital, Ancona; ${ }^{12}$ Radiation Oncology Department, 'A. Gemelli' Hospital, Catholic University of Sacred Heart, Rome; ${ }^{13}$ Radiation Oncology Unit, 'San Raffaele' Hospital, Milan; ${ }^{14}$ Radiation Oncology Unit, ASL TO4, General Hospital, Ivrea; ${ }^{15}$ Radiation Oncology Unit, 'Maggiore della Carità' Hospital, Novara;

${ }^{16}$ Radiation Oncology Unit, Department of Experimental, Diagnostic and Specialty Medicine - DIMES University of Bologna, S. Orsola-Malpighi Hospital, Bologna; ${ }^{17}$ Radiation Oncology Unit, 'SS Annunziata' Hospital, 'G. D'Annunzio' University, Chieti, Italy

Received November 15, 2014; Accepted January 5, 2015

DOI: $10.3892 /$ or.2015.3955

\begin{abstract}
No information is currently available regarding pancreatic cancer (PC) pattern of care in Italy. In the present study, a nationwide survey using a questionnaire was performed to enquire the local standards for PC diagnosis and radiotherapy treatment. Fifty-seven percent of 140 Italian centres completed questionnaire. The main causes of no radiotherapy indication were poor general condition (45\%) and lack of guidelines $(25 \%)$. Physicians $(38 \%)$ employed neoadjuvant therapy in locally advanced PC patients, while in other centres (62\%) adjuvant chemoradiation was administered. Adjuvant gemcitabine-based chemotherapy was selected as the treatment of choice by $59 \%$ of centres. Patients were treated mostly
\end{abstract}

Correspondence to: Dr Gabriella Macchia, Radiation Oncology Unit, Research and Care Foundation 'Giovanni Paolo II', Catholic University of Sacred Heart, Largo A. Gemelli 1, I-86100 Campobasso, Italy

E-mail: gmacchia@rm.unicatt.it

"Joint senior authorship

Key words: pancreatic cancer, radiotherapy, survey, pattern of care, Italy with doses of 50-54.9 Gy on the tumour (or bed) plus lymph nodes. A 3D-CRT technique was used in $81.2 \%$ of centres, while IMRT and IGRT were available in 61.2 and $48.7 \%$ of cases, respectively. Extensive variation exists with regard to patterns of care for PC in Italy. Nevertheless, cooperative studies emerging from this survey appeared beneficial.

\section{Introduction}

Pancreatic cancer (PC) is the fourth leading cause of cancerassociated mortality in the European Union, being the only major cancer site for which no improvement in mortality rates is predicted for both genders. By contrast, a slight increase in mortality rates is expected (1).

Surgical resection is the only curative therapeutic modality with a 5-year postoperative survival rate of $\sim 10-15 \%$. However, only $\sim 20 \%$ of patients are diagnosed with surgically resectable PC, $40 \%$ of patients have metastatic disease and the remaining $40 \%$ have locally advanced $\mathrm{PC}$ in the borderline resectable or unresectable advanced PC form.

Therefore, PC poses a challenge to oncologists and, in particular, controversy surrounds radiation oncology (RO) in the management of PC. Advances in the field of RO have led to improvements in imaging and targeting as well as radiation treatment delivery. Although these advances have the potential 


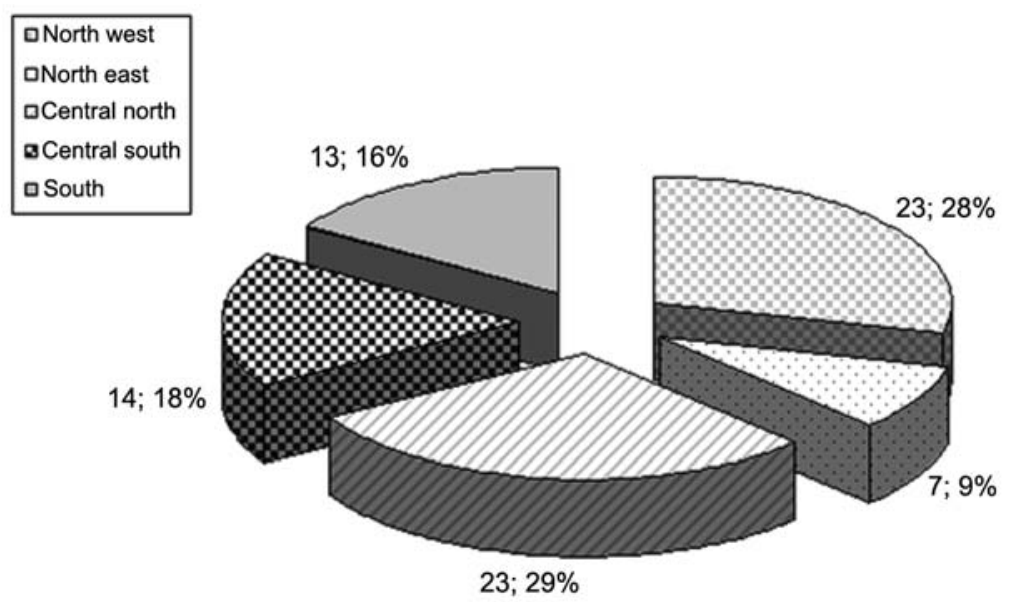

Figure 1. Number and percentages of participating Radiation Oncology Centres according to major Italian geographic areas (North East, North West, Central North, Central South, South and Islands).

to increase tumour control and decrease toxicity, to the best of our knowledge, randomized clinical evidence supporting their widespread application does not exist and there is still a great variety in patterns of practice in different regions worldwide.

Patterns-of-care studies, initially developed in the US in the mid-1970s, are a reliable retrospective study design used to establish the national practice for cancer patients during a specific study period $(2,3)$. Patterns-of-care surveys on PC were conducted in Germany (4), Japan $(5,6)$ and the USA (7). However, to the best of our knowledge, in Italy, no information is available on national RO approach to pancreatic neoplasm.

In the present study, the Italian Society of Radiation Oncology Gastrointestinal Cancer Study Group (AIRO-GI) conducted a nationwide survey among radiation oncologists investigating the clinical practice of PC diagnosis and treatment in Italian university and community hospitals with the aim to subsequently be, proactive in suggesting collaborative multicentre trials. To the best of our knowledge, this is the first report to establish the manner in which $\mathrm{RT}$ is used in the treatment of PC in Italy.

\section{Materials and methods}

Survey design and questionnaire. The study questionnaire was designed by AIRO-GI members in September 2013. The main goal of the questionnaire was to enquire into the local standards for PC diagnosis and RT treatment at the participating centres. Data selected and assessed were from 2012.

The questionnaire, consisting of 40 items, was grouped in four sessions: i) data of the participating physicians: professional site (e.g., university or community hospital, private practice), number and type of PC patients treated per year (2012), work methods (presence or absence of multidisciplinary group), indications for RT and applied techniques [3D conformal RT (3DCRT), intensity-modulated RT (IMRT), image-guided RT (IGRT), intraoperative RT (IORT), stereotactic RT (SBRT), brachytherapy (BRT)]; ii) questions on the local standard diagnostic procedures for PC and issues concerning the histological type; iii) queries concerning the RT treatment standard for resectable (including neoadjuvant and adjuvant therapy) and advanced PC, as well as dose and fractionation details according to treatment modality; iv) questions on RT technical aspect (immobilization systems, use of contrast, gating systems, image fusion), and intention of the individual centres to join multicentre trials. The multiple-choice and open questions were part of the survey.

Participating physicians. The participating centres were not pre-selected. From November 2013 to January 2014 the questionnaire was proposed by the AIRO-GI to all 140 Directors of the Italian Radiation Oncology Institutions as per AIRO website (www.radioterapiaitalia.it: update as per November 2013).

The study methodology was focused on the division of the Italian territory in major geographic areas (North East, North West, North Central, South Central, South and Islands) and the identification for each of them of a radiation oncologist responsible for soliciting and collecting questionnaires via e-mail.

Statistical analysis. Returned questionnaires were collected centrally at the Fondazione 'Giovanni Paolo II'-UCSC, Campobasso and data were entered into an electronic database. The data processing in collaboration with the Institute of Statistics of Aviano occurred in the first six months of 2014. Study data were analyzed by SAS statistical software (version 9.3; SAS Institute Inc., Cary, NC, USA).

\section{Results}

Eighty questionnaires, accounting for $57 \%$ of the 140 Italian centers available on the AIRO website were completed and returned. The responding centres were evenly distributed in five major areas identified with a predominance of the north-central part and reflected the distribution of RT centres throughout the country (Fig. 1).

Session 1. Most of the participating physicians came from community hospitals $(\mathrm{N}=58)$, followed by private $(\mathrm{N}=12)$ and university hospitals $(\mathrm{N}=10)$.

Only 5\% of RT services did not treat PC patients, due to the following reasons: i) patients were referred to a reference centre for pancreatic neoplasm; ii) patients were recorded into 

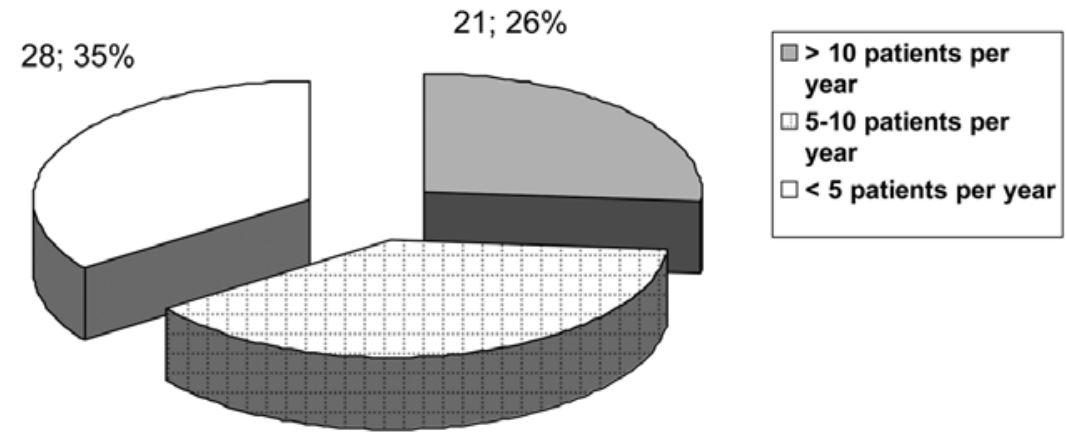

$31 ; 39 \%$

Figure 2. Stratification of participating Radiation Oncology Centres according to the number of treated patients per year (2012).

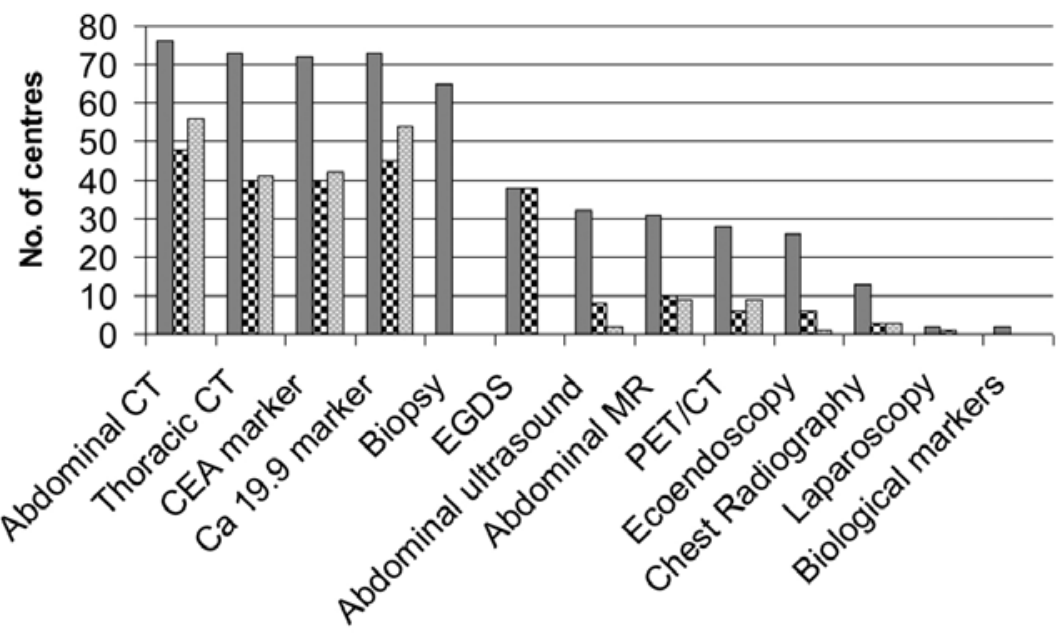

$\square$ Exams required at diagnosis
Exams required at restaging post neoadjuvant treatment
$\square$ Exams required at restaging post adjuvant treatment

Figure 3. Diagnostic examinations required for PC staging (grey bar) and restaging after neoadjuvant (black and white bar) or adjuvant treatment (pale grey bar).

the Hospital (Surgery or Medical Oncology services) but were not addressed to a radiation oncologist.

The majority $(95 \%)$ of radiation oncologists evaluated PC cases. The main causes of no RT indication were poor general condition/co-morbidities (45\%), lack of guidelines $(25 \%)$, inadequate timing (e.g., time elapsed from surgery) (10\%), excessive waiting list (4\%), internal policy (4\%), inadequacies of the centre (e.g., lack of day hospital or inpatient treatment for the management of acute complications) (4\%), patient's age (4\%) and prior systemic therapy (4\%). The absolute figure of PC patients treated in 2012 was 568, although this number did not reflect the reality as only $57 \%$ of Italian centres replied to the questionnaire, and of those only $69 \%$ of centres participating in the survey provided the absolute number of irradiated patients. Stratification in groups according to number of treated patients per year (2012) is shown in Fig. 2. A tumour board for PC was reported by $63 \%$ of participating centres, including 'always' a surgeon, a radiation and a medical oncologist (100\% of cases), often a radiologist $(80 \%)$ and an endoscopyst (80\%), sometimes a nuclear medicine physicist
(36\%), a pathologist (40\%), a physician nutrition specialist (32\%) and a pain therapist (26\%). The tumour board meeting was periodic in approximately two third of cases, while upon request in the the remaining third of cases.

Radical pancreatectomy procedure was mostly performed in the same centre in $59 \%$ of cases, while in other neighbouring health facilities or in Italian referral centres in 23 and $18 \%$ of cases, respectively. IORT was performed exclusively in six centres with $<5$ patients/year in 2012.

Session 2. Fig. 3 shows the diagnostic examinations as required by the different $\mathrm{RO}$ units for $\mathrm{PC}$ staging and restaging following neoadjuvant or adjuvant treatment. In particular, at the time of diagnosis, thoracic and abdominal CT (91 and $95 \%$, respectively) as well as biopsy and marker determination (81 and $91 \%$, respectively) were performed in the majority of patients. Esophageal-gastroduodenoscopy (EGDS), abdominal ultrasound, abdominal magnetic resonance (MR), PET/CT, ultrasound endoscopy and laparoscopy (LPS) were considered less mandatory and use of these techniques was restricted 


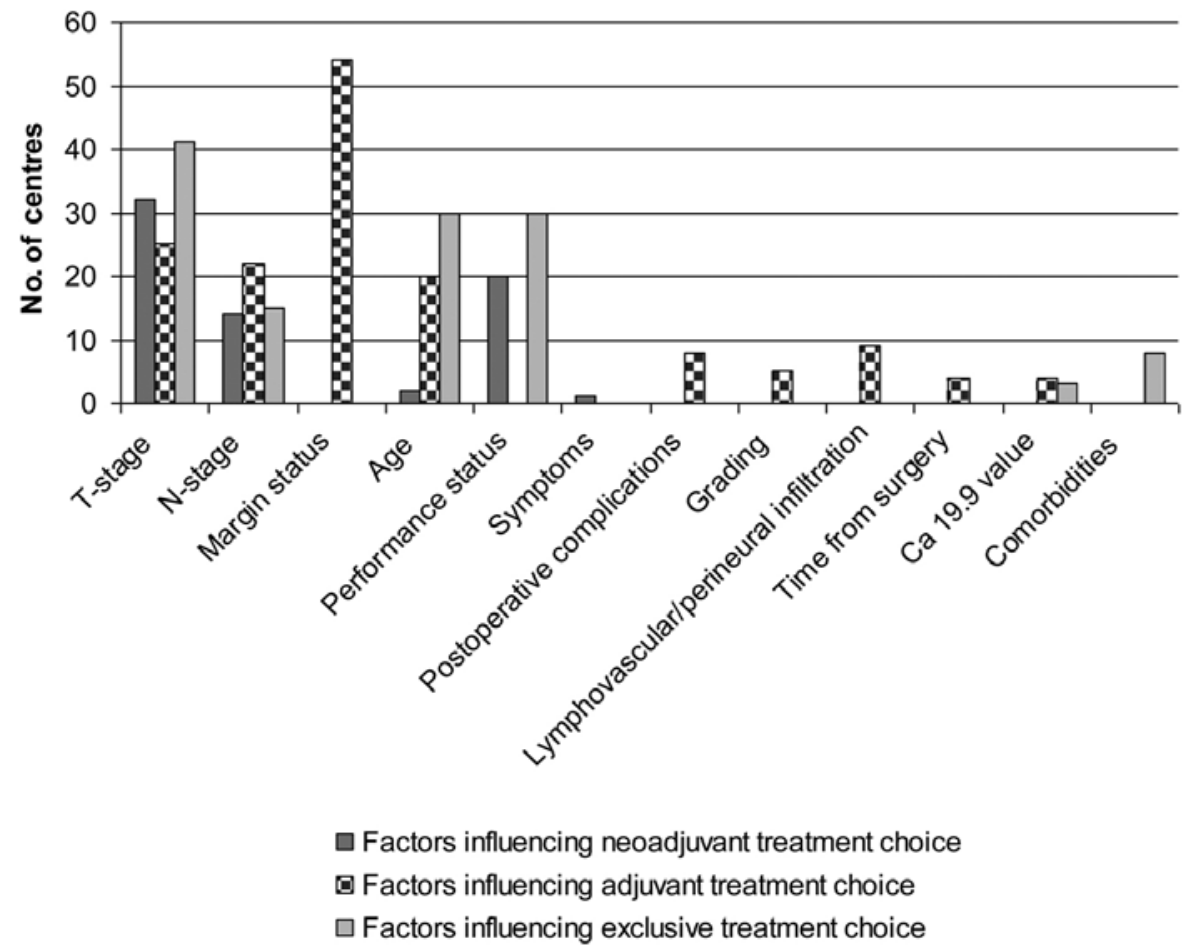

Figure 4. Factors influencing neoadjuvant (grey bar), adjuvant (black and white bar) and exclusive (pale grey bar) treatment.

to selected cases. Chest X-rays and biological markers for examination of polymorphism were not deemed necessary by 79 and $94 \%$ of responding centres, respectively. For the PC treatment, the majority of the physicians $(63 \%)$ needed histological confirmation, although were prompt to perform the treatment only on clinical diagnosis (imaging-markers-symptoms) after a single $(63 \%)$ or at least two $(54 \%)$ biopsy attempts.

Session 3. This session investigated the core of RO approaches in terms of indication criteria, dose, fractionation and chemotherapy schedules. For convenience, this section was divided into three parts while taking into account the three potential approaches (neoadjuvant, adjuvant, exclusive and/or palliative) to the PC from the point of view of the radiooncologist. Clinical factors influencing the treatment approach for the three different settings (neoadjuvant, adjuvant and exclusive) are detailed in Fig. 4.

i) Neoadjuvant setting. This setting account for neoadjuvant RT (nRT), neoadjuvant chemoradiation (nCT/RT) and induction chemotherapy (iCT).

Thirty-eight percent of centres commonly stated the use of nCT/RT, while only $7.5 \%$ in selected cases. Clinical factors influencing the neoadjuvant choice are shown in Fig. 4. PC plus nodal drainage were the contoured target volumes and 45-55 Gy was the range of prescribed doses. Only eight RO services commonly used $>50$ Gy as a total dose. Mostly, fractionation was conventional with 1.8 and $2 \mathrm{~Gy}$ in 70 and $22 \%$, respectively. The drugs used for radiosensitizing were gemcitabine (63.4\%), 5-fluorouracil/capecitabine (26.8\%) and others $(9.8 \%)$.

Induction chemotherapy was performed routinely in $38.7 \%$ of cases and again $7.5 \% \mathrm{RT}$ units selected $\mathrm{iCT}$ in certain cases.
Results of the questionnaire suggested it was possible to indicate $>1$ iCT schedule per centre. The schedules most frequently used were gemcitabine-oxaliplatin $(45 \%)$, gemcitabine alone (32.5\%) or 5-fluorouracil-irinotecan-oxaliplatin (i.e., folfirinox) (12.5\%) mostly with 3 or 4 cycles prior to RT (range, 1-12).

The specialist prescribing and administering chemotherapy drugs was the medical oncologist in $71 \%$ of cases and the radiation oncologist in the remaining $29 \%$.

During a period of 2-8 weeks from the completion of neoadjuvant treatment about two third of centres $(62.5 \%)$ performed clinical instrumental restaging (Fig. 3), mostly after 4 or 6 weeks.

ii) Adjuvant setting. This setting accounted for adjuvant RT (aRT) followed by chemotherapy, adjuvant chemoradiation (aCT/RT) and adjuvant chemotherapy (aCT).

Sixty-two percent of centres declared the use of aCT-RT or aRT followed or not by chemotherapy. The aRT was prescribed to PC bed at doses ranging from 45 to $54 \mathrm{~Gy}$ in $91 \%$ of cases. Doses $>50$ Gy were applied in 19 centres. Nodal drainage in the same dose range was irradiated by $97 \%$ of responders. Almost always conventional fractionation was applied. The drugs most frequently used for radiosensitizing were gemcitabine (58\%) or 5 -fluorouracil/capecitabine (i.v. or per os) $(40 \%)$, although (2\%) a two-drug schedule (gemcitabine plus oxaliplatin) was rarely used. After aRT, 2-4 chemotherapy cycles were added and administered by the majority of centres.

Adjuvant CT was the treatment declared by 47 (58.7\%) of the centres, while $15 \%$ of responders stated use of this technique in selected cases and $26 \%$ of RT units did not perform it in order not to compromise concomitant chemoradiation treatment or due to patient performance status or the decision of the medical oncologist. 


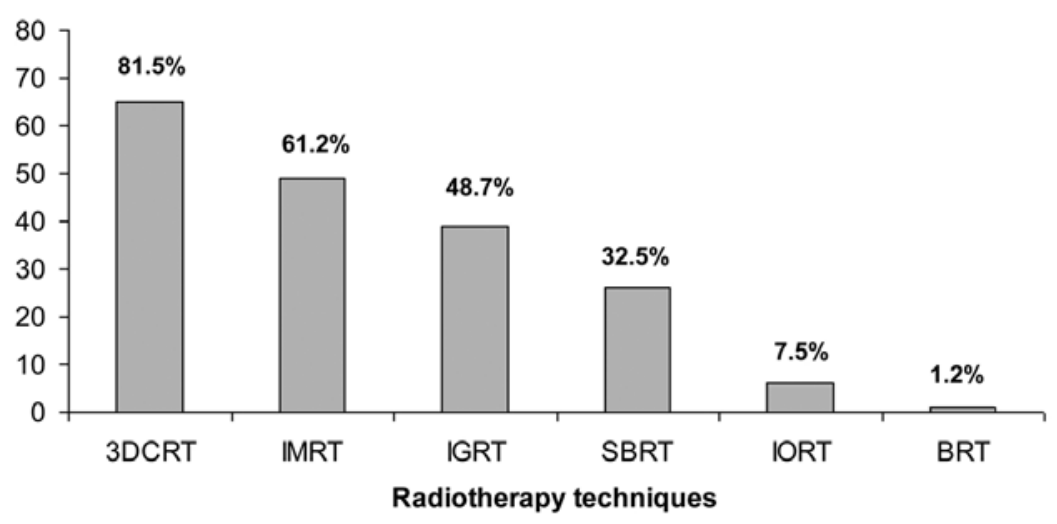

Figure 5. Number of centres using the reported radiotherapy techniques.

The most frequently used drugs were gemcitabine alone $(58.6 \%)$, gemcitabine-oxaliplatin (20.7\%) or 5-fluorouracil/capecitabine (13.8\%) with a number of scheduled cycles ranging from 1 and 10 before RT, although 2, 3 or 6 cycles were the most performed.

Again, the specialist prescribing and administering concomitant chemotherapy was the medical oncologist in $74 \%$ of cases and the radiation oncologist in the remaining $18 \%$. A minority of centres (8\%) did not reply to this question. In the period between 8 and 12 weeks from adjuvant treatment completion, $65 \%$ of centres performed first follow-up (Fig. 3).

iii) Exclusive and palliative setting. This section account for exclusive radiation or chemoradiation (eCT/RT), exclusive chemotherapy (eCT), and palliative radiotherapy (pRT) for the treatment of locally advanced PC unfit for surgery.

Forty-six (57.5\%) and $10(12.5 \%)$ of centres used eCT/RT always or in selected cases, respectively. The dose prescribed to PC ranged between 24 and $66 \mathrm{~Gy}$ in 10-34 fractions, and $57 \mathrm{RO}$ units performed nodal irradiation. The drugs most frequently used as radio sensitizing were gemcitabine alone (56.3\%) or 5-fluorouracil/capecitabine (41.8\%).

Forty-five $(56.25 \%)$ and $8(10 \%)$ of RO units declared the use of eCT in unresectable patients always or in selected cases, respectively. The schemes most frequently used were gemcitabine-oxaliplatin (44.2\%), gemcitabine alone (36.5\%), folfirinox (13.5\%) or 5-fluorouracil/capecitabine (5.8\%) with a number of scheduled cycles ranging from 2 to 12 , although 3 , 4 or 6 were the more frequent numbers of cycles administered.

Forty percent of investigated centres declared the use of pRT, being treated either loco-regional disease (tumour and/or lymph nodes) as well metastases.

Session 4. Treatment techniques for PC irradiation available at censored centres are reported in Fig. 5. The vast majority $(81.5 \%)$ had the possibility to use a 3D-conformal radiotherapy technique. Intensity-modulated radiotherapy and image-guided radiotherapy were available in 61.2 and $48.7 \%$ of cases, respectively. SBRT was an emerging technique and was applied in $32.5 \%$ of RO units.

Immobilization systems were used in $84 \%$ of centres, while a set-up CT scan with intravenous contrast medium was used (always or in selected cases) in $44 \%$ of cases against $56 \%$ not employing it. Six RO units stated the routine use of gating systems against 55 that did not use it, while 15 RO services used gating when the clinical situation required it specifically. Forty-four centres performed routine image fusion in the treatment planning process, while 23 only when the clinical situation specifically required it.

As regards the last question: 'Your centre may be willing to participate in a national study on treatment of PC', most clinicians answered 'yes, of course' (97\%), while a small number $(3 \%)$ refused.

\section{Discussion}

To the best of our knowledge, few studies on PC patterns of RT practice are available (4-7) worldwide and no information is available regarding the Italian reality.

In the present survey, we have focused on the Italian PC radiotherapy practice in 2012, collecting data from 80 (57\%) RO units distributed throughout the country. In comparison with other RO surveys conducted in Italy in 2012 (lung cancer) and 2008 (breast and head/neck cancer), the number of responding centres was encouraging, being 45,48 and $50 \%$, as reported by Ramella et al (8), Aristei et al (9) and Frata et al (10), respectively. This finding reveals an increased sense of membership of radiation oncologists in the RO community and a willingness to deepen their knowledge of the Italian reality in order to improve the quality of their investigation.

The survey attempted to address important issues such as: the main causes of non-indication to radiation treatment, the actual number of patients treated by Italian RO, the preferred or 'mostly chosen' treatment setting and the most applied RT techniques.

Concerning the main causes of non-indication to radiation treatment, our results indicated that slightly less than half of the patients $(45 \%)$ were referred to RT services in poor general condition or carrying severe co-morbidities. This finding should be compared to previous intensive systemic treatments that heavily impacted on patient performance status, and is a bit discordant with the fact that $63 \%$ of the centres participating in the survey reported a tumour board with periodic meeting.

A second cause was the perception of lack of guidelines that was impacted by $25 \%$ of the non-indication to radiation treatment. In fact, the clinical target volume contouring proposal in preoperative/definitive and postoperative PC settings was suggested $(11,12)$. Moreover, AIRO-GI groups published a handy, pocket-sized manual that summarizes the 
main gastrointestinal guidelines [(13) available on line at www. radioterapiaitalia.it/Linee guida AIRO)]. It is likely, however, that the time between these publications and the administration of the survey was not sufficient for their spreading and validation.

The absolute figure of PC patients $(\mathrm{N}=568)$ treated by $\mathrm{RO}$ services in 2012 was low when compared with 11,400 PC esteemed cases in 2012 (14). However, it is to be considered that $\sim 40 \%$ of patients exhibited metastatic disease at diagnosis and underwent chemotherapy or best supportive care. Moreover, a potential bias may lie in the fact that only $57 \%$ of the 140 Italian centres replied to the questionnaire, and of these, only $69 \%$ of centres declared the absolute number of irradiated patients. This figure reflects the changing scenario of international guidelines and the results of randomized trials that have raised questions regarding the optimal treatment of PC. Nevertheless, radiation oncologists have considerable room for improvement in terms of treatment strategies and patterns of RT as well as in terms of combined efforts in prospective studies investigating new combinations of chemotherapy and/ or biologic agents with RT.

The present study also showed the current treatment of patients with PC in Italian hospitals. In a previously conducted meta-analysis the efficacy of neoadjuvant chemoradiation in patients with PC was investigated (15). The authors of that metaanalysis concluded that for patients with resectable tumours, the current data did not suggest an obvious advantage of neoadjuvant therapy. By contrast, patients presenting locally advanced/ unresectable tumours were offered neoadjuvant therapy and then re-evaluated for resection (15). In the Japanese survey, there was no mention of a neoadjuvant approach, although authors reported that $20.8 \%$ of patients were treated using an investigational protocol (6). Our data showed that $\sim 38 \%$ of centres reported the use of $\mathrm{nCT} / \mathrm{RT}$ or $\mathrm{nCT}$ approach. These data are also higher than the German ones. Boeck et al reported a $21 \%$ proportion of neoadjuvant treatment considering the latter unjustified, outside of clinical trials, due to the lack of evidence based rationale for this therapy (4). The discrepancy with international data may be owing to different national guidelines: in Italy, AIOM guidelines suggest that the evaluation of neoadjuvant approach for borderline resectable PC patients (16) should be performed by a tumour board. A second explanation for the relatively high percentage of neoadjuvant treatments is selection bias. PC observed by RO was pre-selected by surgeons and clinical oncologists as potential candidates to irradiation, and this upstream selection justified the high rate of nCT/RT subsequently performed. Furthermore, an incorrect understanding of the so-called term 'neoadjuvant treatment' including operable patients, border-line resectable and locally advanced PC was considered a third possible explanation.

Evidence suggests gemcitabine-oxaliplatin followed by gemcitabine alone were the most employed nCT schedules, administered mostly by 3-4 scheduled cycles (range, 1-12) prior to RT (15). The drug most used for radiosensitizing was gemcitabine (63.4\%), followed by 5-fluorouracil/capecitabine (26.8\%). Andriulli et al (17) assessed the benefit of neoadjuvant/preoperative gemcitabine chemotherapy used alone or in combination with other agents and/or concurrent radiotherapy in patients with localised PC. The results from that metaanalysis provided marginal support regarding the benefits of neoadjuvant/preoperative gemcitabine chemotherapy for patients with localised PC, but indicated a potential advantage for only a minority of those with unresectable lesions. Gemcitabine-based neoadjuvant/preoperative therapies showed a promising rate of tumour response, although at the expense of considerable toxicity (17).

No definite standard has been established in the adjuvant treatment of PC. The available data from randomized phase III trials (ESPAC-1 and CONKO-001) indicate that adjuvant chemotherapy may substantially prolong DFS and cause a moderate increase of overall survival (18-21). However, an optimal chemotherapy regimen remains to be defined. Notably, in Italy as well as in Germany (4), gemcitabine chemotherapy [according to the CONKO-001 trial $(20,21)$ ] was the preferred schema, while aCT according to the 'Mayo regimen' [bolus 5-FU plus folinic acid, ESPAC-1 study (19)] was selected by few physicians. A reduction in toxicity was cited as the explanation, based on the ESPAC-3 trial, whereas gemcitabine was not superior to the Mayo regimen with respect to the primary end-point of overall survival, although the authors reported a $50 \%$ reduction of treatment-associated serious adverse events using gemcitabine (22). In our survey approximately two third of centres reported the use of aCT/RT or aCT approach, with a slight predominance of aCT/RT that again could be explained, considering that centres that adhere to the survey are potentially the most active in the research field and/or the aforementioned upstream patient selection.

Although the optimal RT dose has yet to be defined, the NCCN guidelines have recommended a dose of 50-60 Gy and of 45-54 Gy at conventional fractionation for primary definitive chemoradiation and postoperative RT, respectively (23). Moreover, large outcomes-based analysis for patients treated with adjuvant radiotherapy in resected PC showed that the optimal dose appears to decrease between 50 and $55 \mathrm{~Gy}$ and patients treated within this range had the longest median overall survival (24). In the present survey, most patients were treated with a total dose of 50-54.9 Gy in the exclusive and postoperative groups. Major deviations from these doses were probably associated with the SBRT technique used in 26 centres. Therefore, Italian radiation oncology centres as well as the Japanese ones (6) appear to have appropriatedly utilized the literature guideline recommendations. Of note, no dose data have been reported by German authors (4).

Concerning the RT volumes, in the majority of patients, the primary neoplasm (or tumour bed) plus local drainage were irradiated, irrespective of the treatment setting. Although there is no consensus concerning the elective nodal irradiation (ENI) in PC, it may be justified in a treatment with curative intent due to the high frequency of lymphatic spread (60-80\%) reported in head PC (25-27) and the high rate of local and nodal failure (up to $75 \%$ ) $(28,29)$. Therefore, NCCN practice guidelines have recommended that when 5-FU-based chemoradiotherapy is used, the treatment volumes should include the primary tumour location and the regional lymph nodes (23). Previous reports have indicated that the rate of severe toxicity is greater in patients treated with gemcitabine-based chemoradiotherapy than in those treated with 5-FU-based chemoradiotherapy $(17,30)$. Additional studies investigating the optimal radiation field when using chemotherapy drugs, such as gemcitabine, should be conducted. In particular, 
it is essential in the Italian scenario whereas the drug most frequently used for radiosensitizing was gemcitabine alone $(58 \%)$ or in combination with oxaliplatin (2\%).

With regard to the RT treatment technique, computed tomography-based treatment planning and 3DCRT were used for $>80 \%$ of patients, respectively. This finding is in accordance with the NCCN guidelines (23) and reveal an high quality level of RO procedures.

Moreover, $61 \%$ of PC may be treated by IMRT and $49 \%$ by IGRT. These percentages are noteworthy and suggest that probably PC patients are more likely to be irradiated in technology-advanced structures as compared to less equipped hospitals. Notably, SBRT with or without 3DCRT was frequently reported in Italy. A growing body of literature has contributed to the spread of this latter technique (31-34), which can improve local control by increasing doses without impairing normal tissue. Additional prospective studies are required to assess the efficacy of SBRT in PC.

A main drawback of the present study was the number of responding centres that, although higher than in previous surveys (8-10), limited the analysis. Moreover, we cannot deny heterogeneity in our analysis due to different interpretations of survey queries.

In conclusion, the present study has shown that extensive variation exists with regard to treatment strategies and the patterns of RT. Nevertheless, a favourable attitude towards cooperative studies emerged from the national survey that provided evidence for improved PC treatment in Italy. In the future, repeated surveys and point-by-point comparisons with the results from other countries may demonstrate how RO for PC has been developed and optimized in adhering to the international standard of care.

\section{Acknowledgements}

We are grateful to Professor Massimo Falconi, Professor of 'Vita-Salute San Raffaele' University, Milano, for helping with the drafting of the questionnaire and providential suggestions. Moreover, we are particularly grateful to the 80 leading Italian institutions that kindly participated in the survey. The order of these institutions are listed in alphabetic order: 1. Paola Franzone Radioterapia ASO SSS Antonio e BiagioAlessandria;2.Giovanna Mantello,Radioterapia,State Hospital, Ancona; 3. Fernanda Migliaccio, Radioterapia Oncologica, Ospedale Regionale 'U. Parini', Aosta; 4. Simona Borghesi, Radioterapia, Az.USL 8 Arezzo; 5. Fasano Carla, Radioterapia, Ospedale C. e G. Mazzoni, Ascoli Piceno; 6. Maria Tessa, Radioterapia, Ospedale Cardinal Massaia, Asti; 7. Giovanni Boz, Radioterapia, Centro di Riferimento Oncologico, Aviano; 8. Lisa Paoletti, Radioterapia, Ospedale Santa Maria Annunziata, Bagno a Ripoli (FI); 9. Marco Lioce, Radioterapia, Istituto Tumori 'Giovanni Paolo II', Bari; 10. Francesco Romeo Filippone, Radioterapia, Osp. Papa Giovanni XXIII, Bergamo; 11. Gregorio Moro, Radioterapia, Ospedale degli Infermi, Biella; 12. Renzo Mazzarotto, Radioterapia, Policlinico S. Orsola Malpighi, Bologna; 13. Giovanni Piero Frezza, Radioterapia, Ospedale Bellaria, Bologna; 14. Marco Possanzini, Radioterapia, Ospedale Oncologico Regionale A. Businco, Cagliari; 15. Vincenzo Picardi, Radioterapia, Fondazione 'Giovanni Paolo II', UCSC,
Campobasso; 16. Angelo Tagliagambe, Radioterapia, Ospedale Civico, Carrara; 17. Corrado Spatola, Radioterapia, Policlinico 'G. Rodolico', Catania; 18. Alfio Di Grazia, Radioterapia, REM, Catania; 19. Domenico Genovesi, Radioterapia, Ospedale 'SS Annunziata', Univ. 'G. D'Annunzio', Chieti; 20. Francesca Corazzi, Radioterapia, Città di Castello; 21. Luciano Scandolaro, Radioterapia, Ospedale 'S. Anna', San Fermo della Battaglia (Como); 22. Pierpaolo Ziccarelli, Radioterapia, Ospedale Mariano Santo, Cosenza; 23. Riccardo VignaTaglianti, Radioterapia, A.S.O.S. Croce e Carle, Cuneo; 24. Franco Casamassima, Centro di Radioterapia-Ecomedica, Empoli;25.GiampaoloZini,Radioterapia,Arcispedale S. Anna, Ferrara; 26. Pierluigi Bonomo, Radioterapia, Ospedale Careggi, Firenze; 27. Luca Cionini, Radioterapia, Centro Oncologico Fiorentino, Firenze; 28. Lorenzo Livi, Radioterapia, Casa di Cura S Chiara, Firenze; 29. Almalina Bacigalupo, Radioterapia, AOU IRCCS San Martino, Istituto Nazionale per la Ricerca sul Cancro, Genova; 30. Filippo Grillo Ruggieri, Radioterapia, Ospedale Galliera Genova; 31. Piera Sciacero, Radioterapia, ASL TO4 Ospedale Civile, Ivrea (TO); 32. Ernesto Di Cesare Radioterapia, Ospedale S Salvatore, L'Aquila; 33. Giancarlo Arcangeli, Radioterapia, Ospedale S. Maria Goretti, Latina; 34. Donatella Russo, Radioterapia, Ospedale 'V. Fazzi', Lecce; 35. Rita Bagnoli, Radioterapia, Ospedale Campo Marte, Lucca; 36. Massimo Giannini, Radioterapia, Ospedale Generale Provinciale, Macerata; 37. Antonino Romeo, Radioterapia, IRCCS-IRST Meldola (Forlì-Cesena); 38. Carmela Palazzolo, Radioterapia, AOOR Papardo-Piemonte di Messina, Messina; 39. Mauro Palazzi, Radioterapia, Azienda Ospedaliera Niguarda Ca Granda, Milano; 40. Rita Marina Niespolo, Radioterapia, Ospedale S. Gerardo, Monza; 41. Radioterapia, Centro Aktis Marano di Napoli, Napoli; 42. Paola Murino, Radioterapia, Ospedale Ascalesi Napoli; 43. Milena Di Genesio Pagliuca, Radioterapia, Ospedale Maggiore della Carità, Novara; 44. Luciana Caravatta, Radioterapia, Ospedale S. Francesco, Nuoro; 45. Ornella Lora, Radioterapia, Istituto Oncologico Veneto, Padova; 46. Teresa Cucchiara, Radioterapia, Ospedale Civico, Palermo; 47. Giovanni Battista Ivaldi, Radioterapia, Fondazione Salvatore Maugeri, Pavia; 48. Fabio Arcidiacono, Radioterapia, Ospedale S. Maria della Misericordia, Perugia; 49. Francesca Maurizi, Radioterapia, A.O. Ospedali Riuniti Marche Nord, Pesaro; 50. Aldo Sainato e Luna Valentina Cernusco, Radioterapia, Azienda Ospedaliera Universitaria Pisana, Pisa; 51. Marzano Salvino, Radioterapia, Azienda Ospedaliera ASL 4, Prato; 52. Cinzia Iotti, Radioterapia, Arcispedale di S.M. Nuova, Reggio Emilia; 53. Rossella Maglio, Radioterapia, Ospedale S. Camillo De Lellis, Rieti; 54. Michele Fiore, Radioterapia, Campus Biomedico, Roma; 55. Gian Carlo Mattiucci, Radioterapia, Università Cattolica del Sacro Cuore, Roma; 56. Maria Vittoria Leone, Radioterapia, Ospedale S Giovanni CalibitaFatebenefratelli, Roma; 57. Antonella Ciabattoni, Radioterapia, Ospedale S. Filippo Neri, Roma; 58. Mattia F. Osti, Radioterapia, Ospedale S. Andrea, Roma; 59. Pier Carlo Gentile, Radioterapia, Ospedale Villa S. Pietro FBF, Roma; 60. Nadia Bulzonetti, Radioterapia, Ospedale Umberto I, Roma; 61. Carlo Capirci, Radioterapia, Ospedale Santa Maria della Misericordia, Rovigo; 62. Salvatore Sandro Parisi, Radioterapia, Casa Sollievo della Sofferenza, S Giovanni Rotondo; 63. Marco Orsatti, Radioterapia, Ospedale Civile, 
Sanremo; 64. Corrado Marziano, Radioterapia, Ospedale San Paolo, Savona; 65. Pieromaria Bianchi, Radioterapia, Ospedale Santissima Trinità, Sora; 66. Tindaro Scolaro, Radioterapia, Ospedale Felettino ASL5, La Spezia; 67. Fabrizio Fusconi, Radioterapia, Ospedale Spoleto; 68. Giovanni Silvano, Radioterapia, Ospedale S. Giuseppe Moscati, Taranto; 69. Ernesto Maranzano, Radioterapia, Ospedale S. Maria, Terni; 70. Elena Del Mastro, Radioterapia, IRCCS Candiolo, Torino; 71. Claudia Airaldi, Radioterapia, Ospedale Mauriziano Umberto I $^{\circ}$, Torino; 72. Paolo Rovea, Radioterapia, Ospedale Molinette, Torino; 73. Maria Grazia Ruo Redda, Radioterapia, Ospedale San Luigi Gonzaga, Orbassano (TO); 74. Giuseppe Pani, Radioterapia, Ospedale Santa Chiara, Trento; 75. Vittorio Baggio, Radioterapia, Ospedale S. Maria di Ca' Foncello, Treviso; 76. Sandro Fongione, Radioterapia, Ospedale Santa Maria della Misericordia, Udine; 77. Paolo Antognoni, Radioterapia, Ospedale di Circolo e Fond. Macchi Varese; 78. Maria Chiara Bassi, Radioterapia, Ospedale Castelli, Verbania; 79. Maria Silvia Favretto, Radioterapia, Ospedale San Bortolo, Vicenza; 80. Maria Elena Rosetto, Radioterapia, Ospedale BelColle, Viterbo. The authors would like to thank the two radiation therapy technologists, Angela Palmieri and Laura Palumbo, for their valuable support in collecting and entering data into the database.

\section{References}

1. Malvezzi M, Bertuccio P, Levi F, La Vecchia C and Negri E: European cancer mortality predictions for the year 2013. Ann Oncol 24: 792-800, 2013.

2. Hanks GE, Coia LR and Curry J: Patterns of care studies: past, present, and future. Semin Radiat Oncol 7: 97-100, 1997.

3. Owen JB, Sedransk J and Pajak TF: National averages for process and outcome in radiation oncology: methodology of the patterns of care study. Semin Radiat Oncol 7: 101-107, 1997.

4. Boeck S, Bruns CJ, Sargent M, Schäfer C, Seufferlein T, Jauch KW and Heinemann V: Current oncological treatment of patients with pancreatic cancer in Germany: results from a national survey on behalf of the Arbeitsgemeinschaft Internistische Onkologie and the Chirurgische Arbeitsgemeinschaft Onkologie of the Germany Cancer Society. Oncology 77: 40-48, 2009.

5. Kato H, Usui M, Isaji S, Nagakawa T, Wada K, Unno M, Nakao A, Miyakawa $\mathrm{S}$ and Ohta T: Clinical features and treatment outcome of borderline resectable pancreatic head/body cancer: a multi-institutional survey by the Japanese Society of Pancreatic Surgery. J Hepatobiliary Pancreat Sci: Mar 14, 2013 (Epub ahead of print).

6. Ogawa K, Ito Y, Karasawa K, Ogawa Y, Onishi H, Kazumoto T, Shibuya K, Shibuya H, Okuno Y, Nishino S, Ogo E, Uchida N, Karasawa K, Nemoto K, Nishimura Y; JROSG Working Subgroup of Gastrointestinal Cancers: Patterns of radiotherapy practice for pancreatic cancer in Japan: results of the Japanese Radiation Oncology Study Group (JROSG) survey. Int J Radiat Oncol Biol Phys 77: 743-750, 2010.

7. Wang Y, Schrag D, Brooks GA and Dominici F: National trends in pancreatic cancer outcomes and pattern of care among Medicare beneficiaries, 2000 through 2010. Cancer 120: 1050-1058, 2014.

8. Ramella S, Maranzano E, Frata P, Mantovani C, Lazzari G, Menichelli C, Navarria P, Pergolizzi S and Salvi F: Radiotherapy in Italy for non-small cell lung cancer: patterns of care survey. Tumori 98: 66-78, 2012

9. Aristei C, Amichetti M, Ciocca M, Nardone L, Bertoni F and Vidali C; Italian Society of Radiation Oncology: Radiotherapy in Italy after conservative treatment of early breast cancer. A survey by the Italian Society of Radiation Oncology (AIRO). Tumori 94: 333-334, 2008

10. Frata P, Ponticelli P, Cosentino D, Buffoli A, Di Pilla A, Morrica B and Palazzi M: Radiotherapy resources for the care of head and neck patients in Italy. A survey by the head and neck group of the Italian Association for Radiation Oncology (AIRO). Tumori 94: 59-64, 2008.
11. Caravatta L, Sallustio G, Pacelli F, Padula GD, Deodato F, Macchia G, Massaccesi M, Picardi V, Cilla S, Marinelli A, Cellini N, Valentini V and Morganti AG: Clinical target volume delineation including elective nodal irradiation in preoperative and definitive radiotherapy of pancreatic cancer. Radiat Oncol 7: 86, 2012.

12. Goodman KA, Regine WF, Dawson LA, Ben-Josef E, Haustermans K, Bosch WR, Turian J and Abrams RA: Radiation Therapy Oncology Group consensus panel guidelines for the delineation of the clinical target volume in the postoperative treatment of pancreatic head cancer. Int J Radiation Oncol Biol Phys 83: 901-908, 2012 .

13. Sainato A, Coppola M, Macchia G, Bacigalupo A, Boz G, Sciacero P and Caravatta L: Pancreas - Capitolo 3. In: La Radioterapia dei Tumori Gastrointestinali - Indicazioni e Criteri Guida. Genovesi D (ed), De Paoli A, Macchia G, Sainato A, Lupattelli M, Osti MF, Friso ML, Gambacorta MA, Valvo F, Mantello G, Niespolo R, Dionisi F and Genovesi D (referenti per patologia): Associazione Italiana di Radioterapia Oncologica, pp69-118, 2012.

14. AIRTUM working group: I numeri del cancro in Italia 2012 : Pancreas esocrino: 88-91, 2012.

15. Gillen S, Schuster T, Meyer Zum Büschenfelde C, Friess H and Kleeff J: Preoperative/neoadjuvant therapy in pancreatic cancer: a systematic review and meta-analysis of response and resection percentages. PLoS Med 7: e1000267, 2010.

16. Colucci G, Di Costanzo F, Falconi M, Trodella L, Reni M and Scarpa A: AIOM linee guida, carcinoma del pancreas esocrino, 2013, www.aiom.it. Last access June 20, 2014.

17. Andriulli A, Festa V, Botteri E, Valvano MR, Koch M, Bassi C, Maisonneuve P and Sebastiano PD: Neoadjuvant/preoperative gemcitabine for patients with localized pancreatic cancer: a metaanalysis of prospective studies. Ann Surg Oncol 19: 1644-1662, 2012 .

18. Heinemann V and Boeck S: Perioperative management of pancreatic cancer. Ann Oncol 19 (Suppl 7): vii273-vii278, 2008.

19. Neoptolemos JP, Stocken DD, Friess H, Bassi C, Dunn JA, Hickey H, Beger H, Fernandez-Cruz L, Dervenis C, Lacaine F, Falconi M, Pederzoli P, Pap A, Spooner D, Kerr DJ, Büchler MW; European Study Group for Pancreatic Cancer: A randomized trial of chemoradiotherapy and chemotherapy after resection of pancreatic cancer. N Engl J Med 350: 1200-1210, 2004.

20. Oettle H, Post S, Neuhaus P, Gellert K, Langrehr J, Ridwelski K, Schramm H, Fahlk J, Zuelke C, Burkart C, Gutberlet K, Kettner E, Schmalenberg H, Weigang-Koehler K, Bechstein WO, Niedergethmann M, Schmidt-Wolf I, Roll L, Doerken S and Riess H: Adjuvant chemotherapy with gemcitabine vs observation in patients undergoing curative-intent resection of pancreatic cancer: a randomized controlled trial. JAMA 297: 267-277, 2007.

21. Neuhaus P, Riess H, Post S, Gellert K, Ridwelski K, Schramm H, Zuelke C, Fahlke J, Langrehr J and Oettle H: CONKO-001: final results of the randomized, prospective, multicenter phase III trial of adjuvant chemotherapy with gemcitabine vs. observation in patients with resected pancreatic cancer (PC). J Clin Oncol 26 (Suppl): abs. LBA4504, 2008

22. Neoptolemos JP, Stocken DD, Bassi C, Ghaneh P, Cunningham D, Goldstein D, Padbury R, Moore MJ, Gallinger S, Mariette C, Wente MN, Izbicki JR, Friess H, Lerch MM, Dervenis C, Oláh A, Butturini G, Doi R, Lind PA, Smith D, Valle JW, Palmer DH, Buckels JA, Thompson J, McKay CJ, Rawcliffe CL, Büchler MW; European Study Group for Pancreatic Cancer: Adjuvant chemotherapy with fluorouracil plus folinic acid vs gemcitabine following pancreatic cancer resection: a randomized controlled trial. JAMA 304: 1073-1081, 2010.

23. Pancreatic Adenocarcinoma. v. 2. 2011. National Comprehensive Cancer Network Practice Guidelines. www.ncen.org. Last access June 20, 2014.

24. Hall WA, Colbert LE, Liu Y, Gillespie T, Lipscomb J, Hardy C, Kooby DA, Prabhu RS, Kauh J and Landry JC: The influence of adjuvant radiotherapy dose on overall survival in patients with resected pancreatic adenocarcinoma. Cancer 119: 2350-2357, 2013.

25. Sohn TA, Yeo CJ, Cameron JL,Koniaris L, Kaushal S, Abrams RA, Sauter PK, Coleman J, Hruban RH and Lillemoe KD: Resected adenocarcinoma of the pancreas-616 patients: results, outcomes, and prognostic indicators. J Gastrointest Surg 4: 567-579, 2000.

26. Yoshida T, Matsumoto T, Sasaki A, Shibata K, Aramaki M and Kitano S: Outcome of paraaortic node-positive pancreatic head and bile duct adenocarcinoma. Am J Surg 187: 736-740, 2004.

27. Brunner TB, Merkel S, Grabenbauer GG, Meyer T, Baum U, Papadopoulos T, Sauer R and Hohenberger W: Definition of elective lymphatic target volume in ductal carcinoma of the pancreatic head based on histopathologic analysis. Int J Radiat Oncol Biol Phys 62: 1021-1029, 2005. 
28. Kayahara M, Nagakawa T, Ueno K, Ohta T, Takeda T and Miyazaki I: Lymphatic flow in carcinoma of the distal bile duct based on a clinicopathologic study. Cancer 72: 2112-2117, 1993.

29. Hishinuma S, Ogata Y, Tomikawa M, Ozawa I, Hirabayashi K, Igarashi S: Patterns of recurrence after curative resection of pancreatic cancer, based on autopsy findings. J Gastrointest Surg 10: 511-518, 2006.

30. Crane CH, Abbruzzese JL, Evans DB, Wolff RA, Ballo MT, Delclos M, Milas L, Mason K, Charnsangavej C, Pisters PW, Lee JE, Lenzi R, Vauthey JN, Wong AB, Phan T, Nguyen Q and Janjan NA: Is the therapeutic index better with gemcitabine-based chemoradiation than with 5-fluorouracil-based chemoradiation in locally advanced pancreatic cancer? Int J Radiat Oncol Biol Phys 52: 1293-1302, 2002.

31. Chang DT, Schellenberg D, Shen J, Kim J, Goodman KA, Fisher GA, Ford JM, Desser T, Quon A and Koong AC: Stereotactic radiotherapy for unresectable adenocarcinoma of the pancreas. Cancer 115: 665-672, 2009.
32. Koong AC, Christofferson E, Le QT, Goodman KA, Ho A, Kuo T, Ford JM, Fisher GA, Greco R, Norton J and Yang GP: Phase II study to assess the efficacy of conventionally fractionated radiotherapy followed by a stereotactic radiosurgery boost in patients with locally advanced pancreatic cancer. Int J Radiat Oncol Biol Phys 63: 320-323, 2005.

33. Rwigema JC, Parikh SD, Heron DE, Howell M, Zeh H, Moser AJ, Bahary N, Quinn A and Burton SA: Stereotactic body radiotherapy in the treatment of advanced adenocarcinoma of the pancreas. Am J Clin Oncol 34: 63-69, 2011.

34. Macchia G, Morganti AG, Cilla S, Ippolito E, Massaccesi M, Picardi V, Mattiucci GC, Bonomo P, Tambaro R, Pacelli F, Piermattei A, De Spirito M, Valentini V, Cellini N and Deodato F: Quality of life and toxicity of stereotactic radiotherapy in pancreatic tumours: a case series. Cancer Invest 30: 149-155, 2012. 